\section{NIV for OHS without severe OSAS: is it worth it?}

\author{
P B Murphy, ${ }^{1,2}$ J-P Janssens ${ }^{3}$
}

Home non-invasive ventilation (NIV) has been used to manage chronic respiratory failure since the polio epidemics of the 1940s; however, the support for many of the indications remains based on small non-randomised or uncontrolled studies. Obesity hypoventilation syndrome (OHS) is an increasingly common cause of chronic respiratory failure and indication for home mechanical ventilation; unsurprisingly as the diagnosis remains frequently missed, significant variation exists across Europe. ${ }^{12}$ The majority of patients with OHS have significant obstructive sleep apnoea (OSA) and recent data have demonstrated the benefits of NIV in a randomised controlled trial. ${ }^{3} 4$ However, it has been increasingly clear that the clinical description which was first reported over 60 years ago ${ }^{5}$ is in fact made up of distinct clinical phenotypes, most frequently divided by the nature of the sleepdisordered breathing, which is driving respiratory failure. ${ }^{6}$ In fact, the broad definition is being challenged further with suggestions to incorporate base excess rather than arterial carbon dioxide in the diagnostic criteria and to consider daytime normocapnic subjects with a base excess $\geq 2 \mathrm{mmol} / \mathrm{L}$ as having an early obesity-related nocturnal hypoventilation. ${ }^{7}$ Analogous to the phenotypes being increasingly advocated in COPD, ${ }^{8}$ the subdivisions of OHS may have different risk profiles and require differing treatment strategies.

Masa et $a l^{9}$ present data in this edition of Thorax on treatment responses in this group of chronic obesity-related respiratory failure without severe OSA. The paper provides much information to guide and inform the clinicians in this area. The study enrolled patients from 16 centres that form the 'Spanish NIV network,' an established multicentre network that represents the model for

\footnotetext{
${ }^{1}$ Lane Fox Clinical Respiratory Physiology Research Centre, Guy's \& St Thomas' NHS Foundation Trust, London, UK; ${ }^{2}$ Division of Asthma, Allergy and Lung Biology, King's College London, London, UK; ${ }^{3}$ Division of Pulmonary Diseases, Geneva University Hospital, Geneva, Switzerland

Correspondence to Dr Patrick B Murphy, Lane Fox Respiratory Unit, St Thomas' Hospital, Westminster Bridge Road, London SE1 7EH, UK; patrick.b.murphy@
} kcl.ac.uk future large-scale trials in specialist areas such as home ventilation. Three hundred sixty-five patients were screened, of which $86(24 \%)$ were identified as having chronic obesity-related respiratory failure but without severe OSA, which was defined as an apnoea-hypopnoea index (AHI) of $>30$ events/hour. Earlier descriptions of this phenotype have frequently used an AHI cut-off of $>5$ events/hour leading to previously lower reported prevalence. ${ }^{10}$ The use of the higher cut-off can be supported in that the clinical and health economic impact of treating patients with mildto-moderate OSA with CPAP is less well supported and so can be legitimately withheld. $^{11}$

The data confirm that NIV improves chronic respiratory failure $\left(\mathrm{PaCO}_{2}\right.$ : NIV $\Delta-6 \pm 6 \mathrm{~mm} \mathrm{Hg}$ vs control $\Delta-3 \pm 5 \mathrm{~mm} \mathrm{Hg}$; $\mathrm{p}=0.019)$ and daytime hypersomnolence (Epworth sleepiness scale: NIV $\Delta-3 \pm 4$ vs control $\Delta-1 \pm 3 ; \quad \mathrm{p}=0.021)$ in OHS without OSA to a similar degree to that seen in OHS-OSA supporting current clinical practice. The patient cohort showed high levels of comorbidity and mild-to-moderate respiratory failure $\left(\mathrm{PaCO}_{2}\right.$ : NIV $\left.49 \mathrm{~mm} \mathrm{Hg}\right)$ with a significant female preponderance ( $79 \%$ female) compared with gender split in an unrefined OHS population of approximately $50 \%$. Interestingly, the response to NIV appears to differ from that seen in patients with OSA-OHS from the parallel trial, ${ }^{3}$ with no improvement in weight, lung function or exercise capacity that may have been expected, although patient numbers could have contributed to the failure to demonstrate a significant difference. Given the obvious importance of obesity in the role of OHS, the failure to replicate the weight loss and exercise capacity seen in other patient cohorts raises the possibility that specific focused interventions may be required to achieve this in those patients with OHS without OSA. This phenotyping will allow the clinician to better inform the patient regarding expected benefits of treatment and consider enhanced intervention in treatmentresistant groups, although further clinical trial data to demonstrate a benefit are required.

When evaluating the treatment response seen in the data reported by Masa et al in patients with OHS without severe OSA, it is important to understand the nature of the intervention provided. Patients randomised to NIV received volume-targeted therapy with an initial daytime acclimatisation period followed by a titrated overnight sleep study. Volume-targeted NIV acts to reduce the variability seen during different sleep stages and positions overnight as well as weight change over time. While empirically appealing, the mode has failed to demonstrate a clear clinical advantage over adequately titrated fixed bi-level support. $^{12}$ The reasons for adopting this mode in the trial design are not reported and, although it would be expected that fixed bi-level would produce similar clinical benefits, the data must be viewed with this methodological approach in mind. Furthermore, the limitations of volumetargeted modes of NIV should be well understood with the algorithms appearing more stable and effective when used in a single limb 'intentional leak' configuration rather than with a dual limb or active expiration valve. ${ }^{13}{ }^{14}$ Use of six different home ventilators with different algorithms, and potentially unequal performances when used in autotitrating modes, must also be taken into account. The details of the titration protocol are provided in the online supplement and deviate from previous established practice with changes in pressure support used to ameliorate hypopnoeas and expiratory positive airway pressure titration reserved for the few obstructive apnoeas only. By the nature of the titration protocol an overnight attended respiratory polygraphy was required, this is against the trend in some countries to move towards outpatient setup, often using autotitrating devices. Often, polysomnography is just not available within reasonable time limits for titrating NIV.

Although Masa et al clearly established that NIV in OHS without severe obstructive sleep apnoea syndrome improved arterial blood gases, and serum bicarbonates, its impact on dyspnoea, daytime sleepiness, sleep structure and healthrelated quality of life is either modest or non-significant. Thus, exploring how this phenotype of patients with OHS benefit from NIV in terms of healthcare expenditures, hospital admissions and cardiovascular morbidity is necessary to legitimise our current practice. While there is a high level of cardiovascular comorbidity seen in patients with OHS, this has yet to be demonstrated to be modified by NIV in short trials. ${ }^{4} 15$ In fact, Borel et al $^{4}$ found no significant change in metabolic or 
cardiovascular parameters, pro-inflammatory or anti-inflammatory cytokines or markers of endothelial dysfunction after 1 month of NIV in a randomised trial of unselected OHS subjects. The data reported represent the first stage of a clinically focused trial examining cardiovascular outcomes and healthcare utilisation that will report following a period of 3-year follow-up. Encouragingly, the patients receiving NIV had fewer emergency department visits (emergency room visit days: NIV 0.05 \pm 0.22 vs control $0.23 \pm 0.53$; unadjusted $\mathrm{p}=0.04$ ) during the brief follow-up period ( 2 months), although this significance was lost following adjustment for baseline differences between groups. The results of the long-term follow-up will greatly inform clinical practice in this area and are much needed to guide clinicians and patients.

Competing interests None declared.

Provenance and peer review Commissioned; externally peer reviewed.

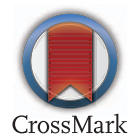

To cite Murphy PB, Janssens J-P. Thorax 2016;71:877-878.

Published Online First 9 August 2016

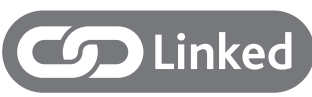

http://dx.doi.org/10.1136/thoraxjnl-2016-208501

Thorax 2016;71:877-878.

doi:10.1136/thoraxjn-2016-208986

\section{REFERENCES}

1 Janssens JP, Derivaz $\mathrm{S}$, Breitenstein $\mathrm{E}$, et al. Changing patterns in long-term noninvasive ventilation. A 7-year prospective study in the Geneva Lake area. Chest 2003;123:67-79.

2 Lloyd-Owen SJ, Donaldson GC, Ambrosino N, et al. Patterns of home mechanical ventilation use in Europe: results from the Eurovent survey. Eur Respir J 2005;25:1025-31.

3 Masa JF, Corral J, Alonso ML, et al. Efficacy of different treatment alternatives for obesity hypoventilation syndrome. Pickwick study. Am J Respir Crit Care Med 2015;192:86-95.

4 Borel JC, Tamisier R, Gonzalez-Bermejo J, et al. Noninvasive ventilation in mild obesity hypoventilation syndrome: a randomized controlled trial. Chest 2012;141:692-702.

5 Auchincloss JH Jr, Cook E, Renzetti AD. Clinical and physiological aspects of a case of obesity, polycythemia and alveolar hypoventilation. J Clin Invest 1955;34:1537-45.

6 Hart N, Mandal S, Manuel A, et al. Rebuttal: 'Obesity hypoventilation syndrome (OHS): does the current definition need revisiting?' Thorax 2014;69:955.
7 Manuel AR, Hart N, Stradling JR. Is a raised bicarbonate, without hypercapnia, part of the physiologic spectrum of obesity-related hypoventilation? Chest 2015;147:362-8.

8 Agusti A, Bel E, Thomas M, et al. Treatable traits: toward precision medicine of chronic airway diseases. Eur Respir J 2016;47:410-19.

9 Masa JF, Corral J, Caballero C, et al. Non-invasive ventilation in obesity hypoventilation syndrome without severe obstructive sleep apnoea. Thorax 2016;71:899-906

10 Kessler R, Chaouat A, Schinkewitch P, et al. The obesity-hypoventilation syndrome revisited: a prospective study of 34 consecutive cases. Chest 2001;120:369-76.

11 Pietzsch JB, Garner A, Cipriano LE, et al. An integrated health-economic analysis of diagnostic and therapeutic strategies in the treatment of moderate-to-severe obstructive sleep apnea. Sleep 2011;34:695-709.

12 Murphy PB, Davidson C, Hind MD, et al. Volume targeted versus pressure support non-invasive ventilation in patients with super obesity and chronic respiratory failure: a randomised controlled trial. Thorax 2012;67:727-34.

13 Carlucci A, Schreiber A, Mattei A, et al. The configuration of bi-level ventilator circuits may affect compensation for non-intentional leaks during volume-targeted ventilation. Intensive Care Med 2013;39:59-65.

14 Khirani S, Louis B, Leroux K, et al. Harms of unintentional leaks during volume targeted pressure support ventilation. Respir Med 2013; 107:1021-9.

15 Borel JC, Roux-Lombard P, Tamisier R, et al. Endothelial dysfunction and specific inflammation in obesity hypoventilation syndrome. PLOS ONE 2009;4: e6733. 